\title{
Venous sinus stenting for reduction of intracranial pressure in IIH: a prospective pilot study
}

\author{
${ }^{*}$ Kenneth C. Liu, MD,,2 Robert M. Starke, MD, MSc, ${ }^{1}$ Christopher R. Durst, MD, ${ }^{2}$ \\ Tony R. Wang, MD, ${ }^{1}$ Dale Ding, MD, ${ }^{1}$ R. Webster Crowley, MD, ${ }^{1,2}$ and Steven A. Newman, MD ${ }^{1,3,4}$ \\ Departments of ${ }^{1}$ Neurological Surgery, ${ }^{2}$ Radiology and Medical Imaging, ${ }^{3}$ Ophthalmology, and ${ }^{4}$ Neurology, University of Virginia, \\ Charlottesville, Virginia
}

\begin{abstract}
OBJECTIVE Idiopathic intracranial hypertension (IIH) may cause blindness due to elevated intracranial pressure (ICP). Venous sinus stenosis has been identified in select patients, leading to stenting as a potential treatment, but its effects on global ICP have not been completely defined. The purpose of this pilot study was to assess the effects of venous sinus stenting on ICP in a small group of patients with $\mathrm{IH}$.

METHODS Ten patients for whom medical therapy had failed were prospectively followed. Ophthalmological examinations were assessed, and patients with venous sinus stenosis on MR angiography proceeded to catheter angiography, venography with assessment of pressure gradient, and ICP monitoring. Patients with elevated ICP measurements and an elevated pressure gradient across the stenosis were treated with stent placement.
\end{abstract}

RESULTS All patients had elevated venous pressure (mean $39.5 \pm 14.9 \mathrm{~mm} \mathrm{Hg}$ ), an elevated gradient across the venous sinus stenosis $(30.0 \pm 13.2 \mathrm{~mm} \mathrm{Hg})$, and elevated ICP $(42.2 \pm 15.9 \mathrm{~mm} \mathrm{Hg})$. Following stent placement, all patients had resolution of the stenosis and gradient $(1 \pm 1 \mathrm{~mm} \mathrm{Hg})$. The ICP values showed an immediate decrease (to a mean of $17.0 \pm 8.3 \mathrm{~mm} \mathrm{Hg}$ ), and further decreased overnight (to a mean of $8 \pm 4.2 \mathrm{~mm} \mathrm{Hg}$ ). All patients had subjective and objective improvement, and all but one improved during follow-up (median 23.4 months; range 15.7-31.6 months). Two patients developed stent-adjacent stenosis; retreatment abolished the stenosis and gradient in both cases. Patients presenting with papilledema had resolution on follow-up funduscopic imaging and optical coherence tomography (OCT) and improvement on visual field testing. Patients presenting with optic atrophy had optic nerve thinning on follow-up OCT, but improved visual fields.

CONCLUSIONS For selected patients with $\mathrm{IH}$ and venous sinus stenosis with an elevated pressure gradient and elevated ICP, venous sinus stenting results in resolution of the venous pressure gradient, reduction in ICP, and functional, neurological, and ophthalmological improvement. As patients are at risk for stent-adjacent stenosis, further follow-up is necessary to determine long-term outcomes and gain an understanding of venous sinus stenosis as a primary or secondary pathological process behind elevated ICP.

https://thejns.org/doi/abs/10.3171/2016.8.JNS16879

KEY WORDS pseudotumor cerebri; idiopathic intracranial hypertension; venous stenosis; stent; pressure gradient; optical coherence tomography; interventional neurosurgery

$\mathrm{I}$ DIOPATHIC intracranial hypertension (IIH) is a syndrome of elevated intracranial pressure (ICP) often of unknown etiology. ${ }^{8,12,18,20}$ The most common symptoms include chronic headaches, pulse-synchronous tinnitus, diplopia, and visual loss. Common associated signs include arcuate visual field defects, papilledema, and abducens nerve palsy. Approximately $10 \%-25 \%$ of patients will develop visual loss as a result of chronic papilledema. ${ }^{1,24}$ A recent randomized clinical trial has found oral acetazolamide to be a beneficial medical therapy in patients with mild $\mathrm{IIH},{ }^{25}$ but acetazolamide is associated with a number of side effects, and a subset of patients are refractory to medical therapy. For patients with medically refractory IIH, optic nerve fenestration and CSF diversion with lumboperitoneal or ventriculoperitoneal shunting are treatment options that may alleviate secondary signs and symptoms without treating the primary pathology. However, these treatment options may be associated with

ABBREVIATIONS BMI = body mass index; ICA = internal carotid artery; ICP = intracranial pressure; $I I H=$ idiopathic intracranial hypertension; $\mathrm{OCT}=$ optical coherence tomography.

SUBMITTED April 8, 2016. ACCEPTED August 5, 2016.

INCLUDE WHEN CITING Published online December 23, 2016; DOI: 10.3171/2016.8.JNS16879.

${ }^{*}$ Drs. Liu and Starke contributed equally to this work. 
significant side effects and disease recurrence and/or progression. ${ }^{24}$

Although many cases of IIH are idiopathic, a number of patients have been found to have venous sinus stenosis as a potential underlying mechanism. During cerebral venography, manometry has demonstrated significant pressure gradients across the stenotic segments with elevated venous pressures most frequently proximally in the superior sagittal sinus. ${ }^{23}$ Currently, it is unclear whether this stenosis is the cause of IIH or the result of elevated ICP as a secondary disease process. Although stenting of the venous sinus stenosis has been shown in select cases to resolve the pressure gradient, ${ }^{2-4,11,14,15,17,19,21-23}$ the effect on ICP remains unclear. Additionally, the effects on visual and neurological outcomes remain incompletely defined. The aims of this prospective pilot study of patients with $\mathrm{IIH}$ and venous sinus stenosis with elevated trans-stenosis pressure gradients are to determine the effects of venous sinus stenting on intravenous pressure, overall ICP, and ophthalmological, neurological, radiographic, and functional outcomes.

\section{Methods}

All patients included in this study were treated in the University of Virginia Health System, and the clinical protocol was approved by the local institutional review board.

The diagnosis of IIH was made based on the updated modified Dandy criteria. ${ }^{13}$ All patients with signs or symptoms attributable to IIH were assessed in the neuro-ophthalmology clinic. Patients whose condition was refractory to medical therapy were evaluated with MRI, angiography, and venography to assess for venous sinus stenosis as well as alternative causes. Patients with evidence of venous sinus stenosis were admitted to the hospital for placement of an ICP monitor, cerebral angiography, venography, and venous pressure manometry and were prospectively followed in our clinical protocol.

\section{Clinical Assessment}

All patients were assessed in the neuro-ophthalmological clinic as well as the neurosurgery clinic.

Signs and symptoms, history of IIH, medical history, physical examination, and prior radiographic examinations were reviewed and recorded. Functional assessment was graded according to the modified Rankin Scale.

\section{Ophthalmic Assessment}

Ophthalmological function was assessed on presentation and during follow-up. Evaluation included visual acuity testing, funduscopic examination, optical coherence tomography (OCT), and visual field testing. Afferent system function was quantitatively assessed with best corrected visual acuity. Automated static perimetry was performed (program 24-2 using a III, or in advanced cases, a V size test object). Funduscopic examination was carried out and images were recorded. OCT of the nerve fiber layer was carried out with the SD (spectral domain) OCT (Cirrus machine). Afferent pupillary defects are also assessed quantitatively using neutral density filters. Studies are performed prior to stent placement to establish diagnosis and to quantify the degree of optic nerve dysfunction. After the procedure, the patients were evaluated with similar techniques.

\section{ICP Monitoring}

Continuous ICP monitoring was carried out following placement of a right frontal ICP monitor. A cerebral CT scan was performed to confirm appropriate positioning and absence of intracranial hemorrhage after placement of the transducer.

\section{Angiography, Venography, and Manometry}

Cerebral angiography, venography, and venous manometry were performed with the patient under minimal conscious sedation. Cerebral angiography was performed in all cases to exclude vascular malformation or fistula as an alternative source of increased ICP. Cerebral angiography was performed with a guide catheter following femoral arterial puncture. Following femoral venous access, a guide catheter was placed into the internal jugular vein below the jugular bulb. The superior sagittal sinus was then selected with a Renegade Hi-Flo microcatheter (inner diameter 0.027 inches, Boston Scientific) over a microwire. After cerebral venography was performed, a pressure transducer was zeroed, and pressure measurements were recorded within the distal, middle, and proximal segments of the superior sagittal sinus, bilateral transverse sinuses, sigmoid sinus, jugular bulb, and cervical internal jugular vein.

\section{Venous Sinus Stenting}

Patients with a diagnosis of IIH, significant pressure gradient across a venous sinus stenosis, and elevated intracranial pressure were offered venous sinus stenting. Patients with no evidence of elevated ICP and significant venous pressure gradient were not offered endovascular stenting, regardless of the presence of angiographic venous sinus stenosis. Prior to stent placement, patients were given loading doses of aspirin $(325 \mathrm{mg})$ and clopidogrel (600 mg). Therapeutic P2Y12 (<208) and ARU (620-672) levels were assessed as early as 6 hours after administration, and additional clopidogrel and aspirin were then administered as necessary.

After induction of general anesthesia and endotracheal intubation, arterial and venous femoral access was obtained. An activated clotting time of 250-300 seconds was maintained through intravenous administration of heparin. After venous access was obtained, a support catheter was placed into the internal jugular vein or sigmoid sinus. Pressure assessments were then confirmed across the venous sinus stenosis, and venous sinus stenting was carried out with an appropriately sized device (Precise [Cordis, $\mathrm{n}$ = 4], Protégé [Covidien, $\mathrm{n}=6$ ], Wallstent [Boston Scientific, $n=2]$ ). After angiographic confirmation of appropriate stent placement, pressure measurements were again assessed. Poststenting arteriograms were obtained to look for changes in hemispheric venous drainage patterns. ICP monitoring was performed continuously throughout the procedure. 
TABLE 1. Summary of demographic and clinical characteristics of study participants

\begin{tabular}{|c|c|c|c|c|c|c|c|c|c|}
\hline \multirow[b]{2}{*}{$\begin{array}{l}\text { Case } \\
\text { No. }\end{array}$} & \multirow{2}{*}{$\begin{array}{c}\text { Age } \\
\text { (yrs), } \\
\text { Sex }\end{array}$} & \multirow[b]{2}{*}{$\begin{array}{c}\text { BMl } \\
\left(\mathrm{kg} / \mathrm{m}^{2}\right)\end{array}$} & \multicolumn{4}{|c|}{ Before Stent Placement } & \multicolumn{3}{|c|}{ After Stent Placement } \\
\hline & & & $\begin{array}{c}\text { Max Pre-Stenosis } \\
\text { Pressure }\end{array}$ & $\begin{array}{c}\text { Max Post-Stenosis } \\
\text { Pressure }\end{array}$ & $\begin{array}{l}\text { Pre-Stenting } \\
\text { ICP Range }\end{array}$ & $\begin{array}{c}\text { Immediate } \\
\text { Pre-Stenting ICP }\end{array}$ & $\begin{array}{l}\text { Venous } \\
\text { Gradient }\end{array}$ & $\begin{array}{c}\text { Immediate } \\
\text { Post-Stenting ICP }\end{array}$ & $\begin{array}{l}\text { POD 1, ICP } \\
\text { Range }\end{array}$ \\
\hline 1 & $41, \mathrm{~F}$ & 40.3 & 30 & 7 & $20-50$ & 30 & 1 & 17 & $8-19$ \\
\hline 2 & $25, M$ & 39.2 & 46 & 9 & $30-55$ & 45 & 1 & 14 & $2-16$ \\
\hline 3 & $18, \mathrm{~F}$ & 58.1 & 38 & 5 & $25-40$ & 37 & 3 & 17 & $5-18$ \\
\hline 4 & $48, F$ & 35.2 & 28 & 14 & $25-34$ & 25 & 0 & 8 & $0-4$ \\
\hline 5 & $27, \mathrm{~F}$ & 46.2 & 37 & 11 & $26-47$ & 45 & 0 & 22 & $8-17$ \\
\hline 6 & $28, F$ & 56.4 & 68 & 22 & $33-73$ & 50 & 0 & 24 & $10-20$ \\
\hline 7 & $28, F$ & 34.3 & 61 & 7 & $44-61$ & 45 & 1 & 11 & $10-20$ \\
\hline 8 & $35, F$ & 25.1 & 22 & 8 & $25-40$ & 27 & 1 & 19 & $9-19$ \\
\hline 9 & $59, F$ & 38.9 & 28 & 10 & $30-35$ & 34 & 0 & 14 & $6-16$ \\
\hline 10 & $33, F$ & 41.4 & 37 & 2 & $25-36$ & 36 & 4 & 24 & $9-17$ \\
\hline
\end{tabular}

$\mathrm{POD}=$ postoperative day.

\section{Clinical and Radiographic Follow-Up}

Follow-up was scheduled for the neurosurgery and neuro-ophthalmology clinics 3 months after stenting and then annually thereafter. Radiographic follow-up was performed with cerebral angiography and venography at 3 and 12 months after stenting with assessment of the venous pressure gradient across the stent. Patients were then followed annually with CT angiography and venography with repeat cerebral angiography, venography, and venous manometry as needed. All adjudicated outcomes were defined by a neuro-ophthalmologist not involved in the stenting procedure.

\section{Statistical Analysis}

Data are presented as median and range for continuous variables and as frequency for categorical variables. Statistical analyses of categorical variables was performed using chi-square and Fisher's exact tests as appropriate. Comparison of means was performed with the Wilcoxon rank-sum test. A p value $\leq 0.05$ was considered statistically significant.

\section{Results}

\section{Patient and IIH Characteristics}

A total of 10 patients with medically refractory IIH were determined to be appropriate candidates for venous stenting. The patients' mean age at the time of treatment was $34.1 \pm 12.3$ years (range 17-59 years, Table 1). Nine of the 10 patients were women. The mean body mass index (BMI) was $41.5 \pm 9.8 \mathrm{~kg} / \mathrm{m}^{2}$ (range $25.1-56.4 \mathrm{~kg} / \mathrm{m}^{2}$ ), and 9 patients had a BMI greater than $30 \mathrm{~kg} / \mathrm{m}^{2}$. In all 10 cases, medical therapy for IIH had failed, as evidenced by chronic severe headaches and visual decline. Additionally, 5 of 10 patients had pulse-synchronous tinnitus. Four of 10 patients had a history of prior treatment (lumboperitoneal shunt, ventriculoperitoneal shunt, or optic nerve sheath fenestration) with incomplete resolution of their symptoms. All patients had a history of elevated pressure on lumbar puncture on prior workup (mean $42.5 \pm 11.2 \mathrm{~cm}$ $\mathrm{H}_{2} \mathrm{O}$, range $27-55 \mathrm{~cm} \mathrm{H}_{2} \mathrm{O}$ ). All patients presented with visual field abnormalities on formal testing, 7 patients had papilledema on funduscopic and OCT testing, and 3 patients had optic nerve atrophy (Table 2).

\section{Cerebral Venography and Stenting}

Nine of 10 patients had bilateral venous stenosis involving the transverse sinus, and 1 patient had unilateral stenosis with contralateral hypoplasia. The maximum venous pressure was elevated in all patients (mean $39.5 \pm 14.9 \mathrm{~mm}$ $\mathrm{Hg}$, range 22-68 $\mathrm{mm} \mathrm{Hg}$, Table 1). All patients had an elevated pressure gradient across the stenosis (mean 30.0 $\pm 13.2 \mathrm{~mm} \mathrm{Hg}$, range $14-52 \mathrm{~mm} \mathrm{Hg}$ ). ICP was elevated in all patients and paralleled venous pressures (mean $42.2 \pm$ $15.9 \mathrm{~mm} \mathrm{Hg}$, range $20-73 \mathrm{~mm} \mathrm{Hg}$ ).

All patients were treated with unilateral stent placement. Following stenting, all patients had significant angiographic improvement of the stenosis with concomitant resolution of the pressure gradient (mean $1 \pm 1 \mathrm{~mm} \mathrm{Hg}$, range 0-4). All patients had a decrease in ICP immediately following stenting (to a mean of $17.0 \pm 8.3 \mathrm{~mm} \mathrm{Hg}$, range $0-24 \mathrm{~mm} \mathrm{Hg}$ ) and additional reduction overnight (to a mean of $8 \pm 4.2 \mathrm{~mm} \mathrm{Hg}$, range $0-20$ ).

\section{Clinical Outcomes}

Patients were assessed with neurological, ophthalmological, and radiographic follow-up. The median duration of clinical follow-up was 23.5 months (mean 22.4 months, range 15.7-31.6. months). No patients had peri- or postprocedural complications from stenting. One patient had patchy hair loss despite a limited radiation dose; this may have been attributable to taking clopidogrel. Functional status was assessed through the modified Rankin Scale prior to stenting, prior to discharge, and on follow-up (Fig. 1). All but one patient had objective and subjective improvement during follow-up. One patient initially had clinical improvement, but then had a decline in clinical condition. Repeat imaging demonstrated adjacent-stent stenosis, and repeat stenting resulted in clinical improvement. Nine of 10 patients reported subjective improvements in headaches. One patient reported improvement at 
TABLE 2. Baseline and post-stenting follow-up ophthalmological examination findings

\begin{tabular}{|c|c|c|c|c|c|c|c|c|}
\hline \multirow{2}{*}{$\begin{array}{l}\text { Case } \\
\text { No. }\end{array}$} & \multicolumn{2}{|c|}{ Papilledema } & \multicolumn{2}{|c|}{ Acuity } & \multicolumn{2}{|c|}{ OCT } & \multicolumn{2}{|l|}{ Visual Fields } \\
\hline & Baseline & Follow-Up & Baseline & Follow-Up & Baseline & Follow-Up & Baseline & Follow-Up \\
\hline 1 & No & No & $20 / 20,20 / 40$ & $\begin{array}{l}20 / 20,20 / 30 \\
\text { (improved) }\end{array}$ & $43 / 55$ & $47 / 58$ & $\begin{array}{l}\text { Nasal constriction, OD; only tiny island } \\
\text { vision temporally, OS }\end{array}$ & $\begin{array}{l}\text { Mild improve- } \\
\text { ment }\end{array}$ \\
\hline 2 & Yes & No & $20 / 20$ & $20 / 20$ & $422 / 410$ & $105 / 55$ & $\begin{array}{l}\text { Enlarged blind spot, OD; inf/sup arcuate } \\
\text { defects, severe constrictions, OS }\end{array}$ & Improvement \\
\hline 3 & Yes & No & $20 / 400,20 / 20$ & $\begin{array}{r}20 / 100,20 / 20 \\
\text { (improved) }\end{array}$ & $358 / 300$ & $85 / 77$ & $\begin{array}{l}\text { Dense sup/inf arcuate defects, OD; mild } \\
\text { sup/inf arcuate defects, OS }\end{array}$ & Improvement \\
\hline 4 & Yes & No & $20 / 30,20 / 25$ & $\begin{array}{l}20 / 25,20 / 25 \\
\quad \text { (improved) }\end{array}$ & $184 / 208$ & $86 / 85$ & $\begin{array}{l}\text { Moderate inf arcuate defect, OD; mild inf } \\
\text { arcuate defect, OS }\end{array}$ & Improvement \\
\hline 5 & Yes & No & $20 / 30,20 / 200$ & $20 / 30,6 / 200$ & $244 / 242$ & $42 / 54$ & Inf/sup arcuate defects, OS > OD & Improvement \\
\hline 6 & Yes & No & $8 / 200,7 / 200$ & $\begin{array}{l}\text { 20/200 (im- } \\
\text { proved) }\end{array}$ & $157 / 225$ & $62 / 65$ & Sup/inf arcuate defects, OD; NLP, OS & $\begin{array}{l}\text { Mild improve- } \\
\text { ment }\end{array}$ \\
\hline 7 & Yes & No & $20 / 100,20 / 80$ & $\begin{array}{l}\text { 20/20, } 20 / 25 \\
\quad \text { (improved) }\end{array}$ & $196 / 296$ & $81 / 98$ & Sup/inf arcuate defects, OU & Improvement \\
\hline 8 & No & No & $20 / 20$ & $20 / 20$ & $60 / 56$ & $54 / 50$ & $\begin{array}{l}\text { Inferior arcuate defects, OD; superior and } \\
\text { inferior arcuate, OS }\end{array}$ & Improvement \\
\hline 9 & Yes & No & $20 / 20,20 / 60$ & $20 / 20,20 / 60$ & $212 / 158$ & $104 / 78$ & $\begin{array}{l}\text { Enlargement of blind spot w/ inf }>\text { sup arcu- } \\
\text { ate defects, OD; sup \& inf arcuate OS }\end{array}$ & Improvement \\
\hline 10 & No & No & $20 / 30,20 / 20$ & $20 / 25,20 / 20$ & $47 / 56$ & & $\begin{array}{l}\text { Mild depression w/ sup > inf arcuate defect, } \\
\text { OD; possible mild nasal step, OS }\end{array}$ & Improvement \\
\hline
\end{tabular}

inf = inferior; NLP = no light perception; OCT = optical coherence tomography; OD = oculus dexter (right eye); OS = oculus sinister (left eye); OU = oculus uterque (both eyes); sup = superior.

the 14-month follow-up visit, but at the 25-month followup visit reported that the headache frequency and severity was unchanged from baseline (before stenting). The patient had both subjective and objective improvement with respect to ophthalmological outcomes.

\section{Radiographic Outcomes}

Before stent placement, the median percentage stenosis was $71.4 \%$ (median $74.2 \%$, range $60.0 \%-88.5 \%$ ). Immediately after stent placement, the diameter of the treated area increased to between 1.9 and 6.5 times the pre-stenting value (median 3.5 times the pre-stent diameter, mean 3.7 times the pre-stent diameter). All patients had cerebral angiography, venography, and assessment of venous pressures at a minimum of 3 months after stent placement and again at a minimum of 1 year after stent placement. The median duration of radiographic follow-up was 15.5 months (mean 14.2 months, range 12-29.3). Eight patients had no significant in-stent or stent-adjacent stenosis $(<$ $10 \%$ ). On 3-month follow-up cerebral venography, 2 patients had stent-adjacent stenosis. One of these 2 patients (mentioned above under Clinical Outcomes) had experienced a return of her symptoms and had a maximum pressure of $28 \mathrm{~mm} \mathrm{Hg}$ and a new pressure gradient of 15 $\mathrm{mm} \mathrm{Hg}$ with $75 \%$ stent-adjacent stenosis. Repeat stenting resulted in resolution of the pressure gradient and the patient's symptoms, and follow-up imaging demonstrated no significant stenosis. The other patient had experienced an improvement in symptoms without complete resolution. At 3-month follow-up angiography, the maximum venous pressure was $68 \mathrm{~mm} \mathrm{Hg}$ with a gradient of $46 \mathrm{~mm} \mathrm{Hg}$ and
$65 \%$ stent-adjacent stenosis. This was treated with repeat stenting, which resulted in resolution of the pressure gradient and clinical improvement; follow-up imaging demonstrated no significant stenosis. The remaining patients did not have significant stenosis on follow-up and had a mean pressure gradient across the stent of $4 \pm 2.5 \mathrm{~mm} \mathrm{Hg}$ (range 1-7 mm Hg).

\section{Ophthalmology Outcomes}

Overall ophthalmic follow-up occurred at a median of 16.5 months (mean 18.0 months; range 2.1-31.6 months). No patient had worsening of visual acuity following stenting (Table 2). All 7 patients presenting with papilledema had resolution as demonstrated on funduscopic imaging and OCT and improvement on formal visual field testing. All 3 patients presenting with optic atrophy were noted to have optic nerve thinning on follow-up OCT but had improvement in visual field testing. In the 2 cases in which repeat stenting was performed, one patient demonstrated no change in visual acuity, but improvement in papilledema, OCT, and visual fields assessment on neuroophthalmological follow-up. In the second patient, neuroophthalmological follow-up demonstrated improvement in visual acuity, papilledema, OCT, and mild improvement in visual fields on follow-after repeat stenting.

\section{Illustrative Case}

This patient (Case 2) with a history of morbid obesity, chronic headaches, and a positive family history of IIH presented with worsening headaches and a decline in vi- 


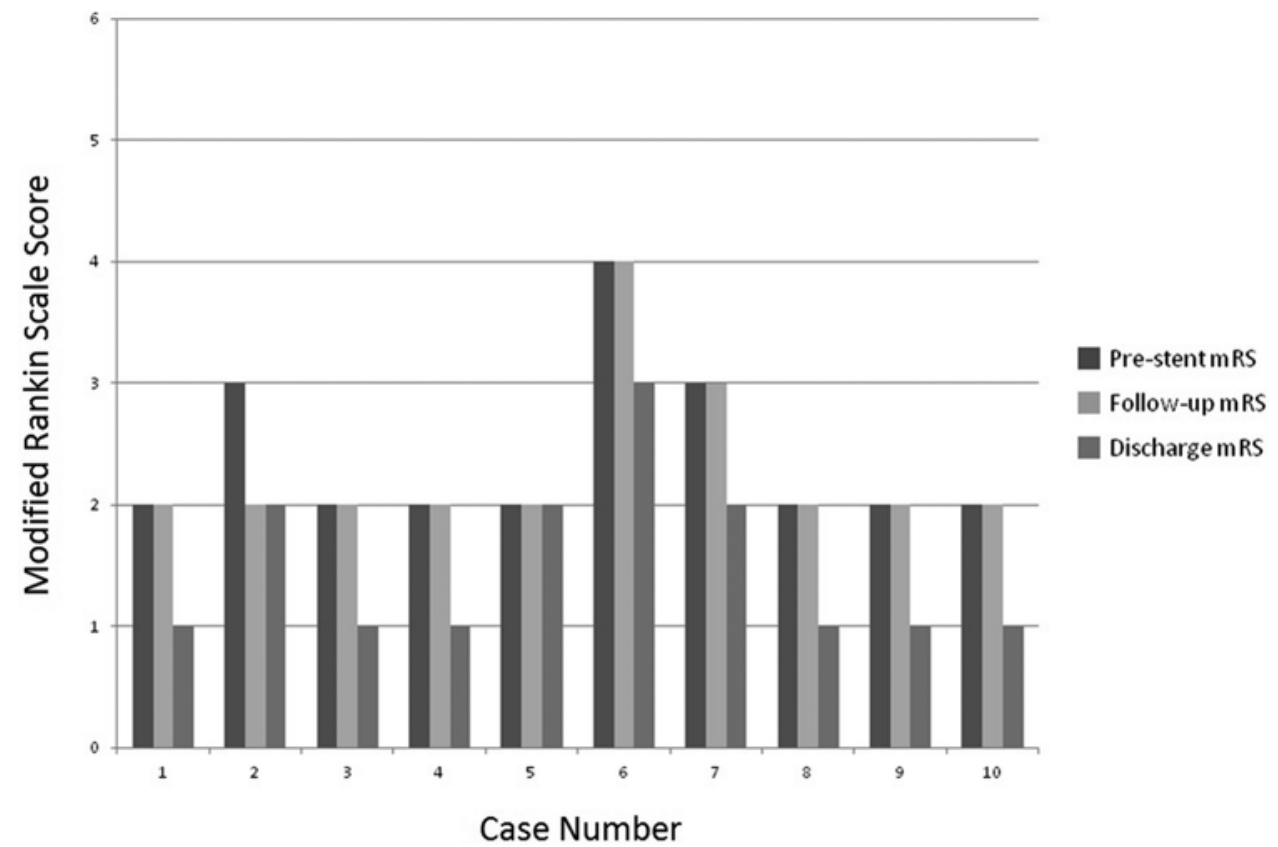

FIG. 1. Functional outcomes as assessed by means of the modified Rankin Scale (mRS) prior to stenting, prior to discharge, and on follow-up.

sion. Funduscopic examination demonstrated papilledema (Fig. 2A); visual field testing confirmed bilateral arcuate defects (Fig. 2B); and OCT displayed marked nerve fiber layer thickening (Fig. 2C). MRI (T2 and T1 with contrast) demonstrated distension of the perioptic subarachnoid spaces bilaterally (Fig. 3A) and an empty sella consistent with intracranial hypertension. Lumbar puncture revealed an opening pressure of $36 \mathrm{~cm} \mathrm{H}_{2} \mathrm{O}$, and the patient experienced temporary improvement after high-volume release of CSF. IIH was diagnosed after further workup demonstrated no other etiology, and acetazolamide treatment was initiated. Initially, the patient's condition improved with medical therapy, but he then had further visual decline. MR angiography demonstrated bilateral transverse sinus stenosis, and cerebral angiography demonstrated prominent focal stenosis of the right transverse sinus (solid arrows, Fig. 3B) and stenosis with diminished flow through the left transverse sinus (dashed arrow, Fig. 3B). The patient was admitted to the hospital, and an ICP monitor was placed. Over 24 hours, the ICP measurements ranged from
23 to $55 \mathrm{~mm} \mathrm{Hg}$. Intravenous pressure monitoring demonstrating a mean venous pressure of $46 \mathrm{~mm} \mathrm{Hg}$ proximal to the stenosis in the superior sagittal sinus (Fig. 3C) and transverse sinuses, with a precipitous decrease to $9 \mathrm{~mm}$ $\mathrm{Hg}$ just distal to the stenosis in the sigmoid sinus. The patient was started on aspirin and clopidogrel and underwent stenting of the dominant right transverse sinus (Fig. 4A), which abolished the pressure gradient and resulted in a decrease of the ICP to $14 \mathrm{~mm} \mathrm{Hg}$. Overnight ICP monitoring demonstrated a mean ICP of $3 \mathrm{~mm} \mathrm{Hg}$ (range 2-16 mm Hg; Fig. 4B). Follow-up cerebral venography (Fig. 4C) and angiography (Fig. 4D) both demonstrated stent patency. The patient had improvement of his headaches and vision. Funduscopic examination demonstrated resolution of papilledema (Fig. 5A). Visual field examination demonstrated bilateral improvement (Fig. 5C), and OCT demonstrated resolution of nerve fiber thickening (Fig. 5B).

\section{Discussion}

In a significant number of cases, the best medical thera-
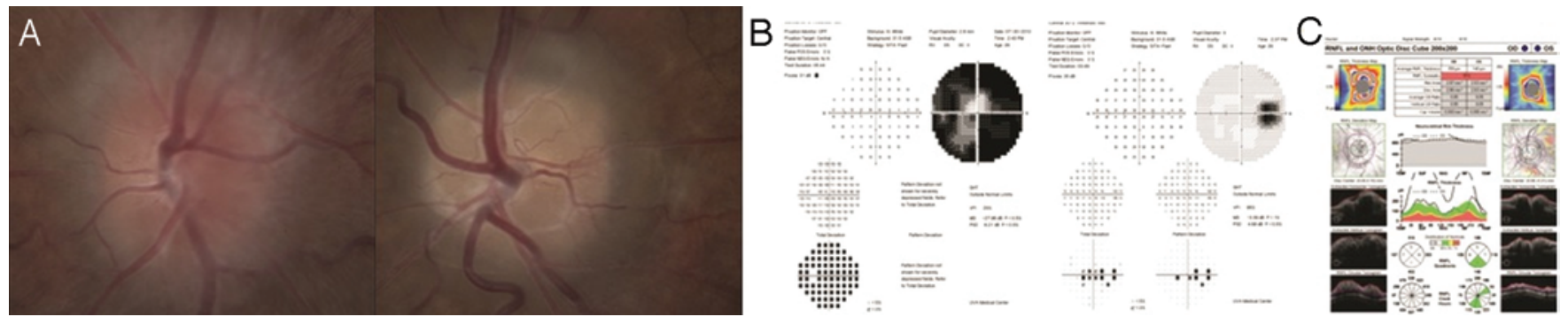

FIG. 2. Case 2. Preoperative ophthalmological examination findings. A: Images from preoperative funduscopic examination showing papilledema. B: Results of visual field testing showing bilateral arcuate defects. C: Results of optical coherence tomography showing marked nerve fiber layer thickening. Figure is available in color online only. 

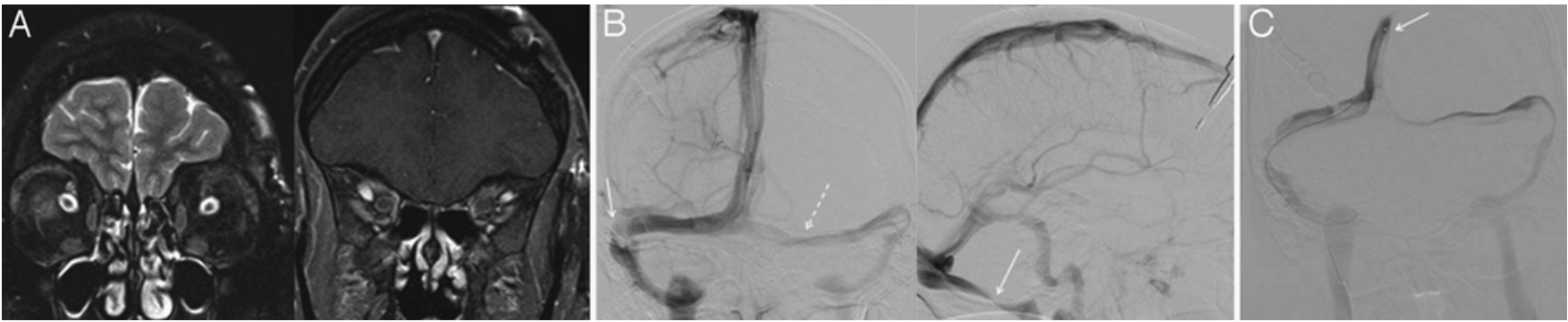

FIG. 3. Case 2. Preoperative imaging studies. A: Preoperative coronal T2-weighted (left) and Gd-enhanced T1-weighted (right) MR images showing distension of the perioptic subarachnoid spaces and an empty sella. B: Cerebral angiography. Anteroposterior (AP) (left) and lateral (right) views of a right internal carotid artery (ICA) injection in the venous phase showing prominent focal stenosis of the right transverse sinus (solid arrows) and stenosis of the left transverse sinus, with diminished flows (dashed arrow). C: Cerebral venography. AP view of a superior sagittal sinus injection, performed during venous manometry, which showed a trans-stenosis pressure gradient of $37 \mathrm{~mm} \mathrm{Hg}$ (mean venous pressures of 46 and $9 \mathrm{~mm} \mathrm{Hg}$ proximal and distal to the stenosis, respectively).

py will not provide sufficient control of IIH, and the patients will continue to have progressive visual loss. ${ }^{1,24}$ Treatments including optic nerve sheath fenestration and CSF shunting may help alleviate signs and symptoms, but they often do not provide durable or complete relief and may be associated with additional side effects. ${ }^{16,18,24}$ In this prospective pilot study, patients with medically refractory IIH and venous sinus stenosis with a trans-stenosis venous pressure gradient underwent venous sinus stenting with concurrent ICP monitoring. The novel findings of this study are that in patients with IIH, elevated ICP, and venous stenosis with an elevated pressure gradient, venous sinus stenting resulted in 1) immediate resolution of the venous pressure gradient across the stenosis; 2) significant decrease in ICP values to the normal range; 3 ) improvements in functional outcomes and neurological examinations; and 4) improvements in ophthalmological examination findings. Specifically, patients presenting with papilledema had resolution as demonstrated on funduscopic imaging and OCT and improvement on visual field testing, and patients presenting with optic atrophy had optic nerve thinning on followup OCT, but improvement in visual field testing.
In all patients, venous stenting resulted in immediate resolution of the venous pressure gradient. Additionally, this is the first study to consistently demonstrate that venous stenting results in a definitive decrease in ICP within 24 hours of treatment. This has previously been inferred through assessments of pressure in the CSF system in patients undergoing lumbar puncture following stenting, but a formal prospective study has not been previously performed ${ }^{23}$ In the current study, the ICP values were elevated in parallel with the venous pressure values. Currently, there are no guidelines for stenting based on the venous pressure gradient, but a pressure gradient as low as $8 \mathrm{~mm}$ $\mathrm{Hg}$ has been proposed as a threshold. ${ }^{2}$ We recommend that patients with IIH refractory to medical therapy, venous sinus stenosis, and a venous pressure gradient receive treatment with venous sinus stenting when there are significant signs and symptoms and when their ICP is found to be elevated through assessment with an ICP monitor or lumbar puncture. Further assessment is needed to develop optimal guidelines for treatment.

This is the first prospective study of venous sinus stenting for IIH that has employed a rigorous set of indepen-
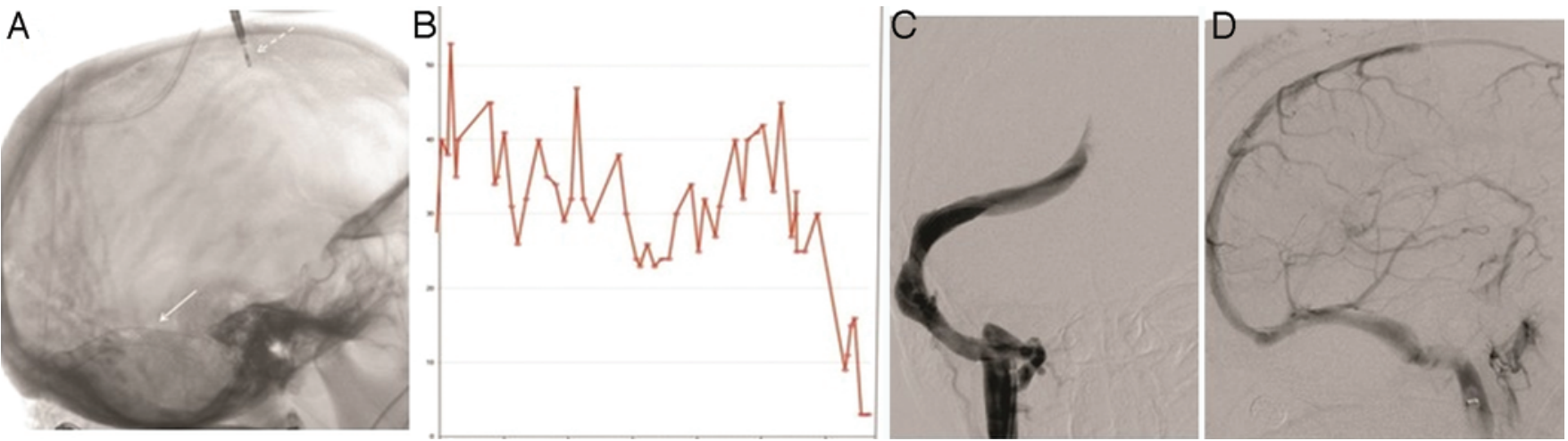

FIG. 4. Case 2. Postoperative studies. A: Angiogram (unsubtracted view) obtained after intraparenchymal ICP monitor placement (dashed arrow) and endovascular stenting of the dominant right transverse sinus (solid arrow). B: Results of ICP monitoring showing a reduction of the preoperative ICP $(23-55 \mathrm{~mm} \mathrm{Hg}$ over a 24-hour period) to $14 \mathrm{~mm} \mathrm{Hg}$ immediately after stenting and then to a mean ICP of $3 \mathrm{~mm} \mathrm{Hg}$ the next day (range 2-16 mm Hg). C and D: Images from follow-up cerebral venography, AP view of a superior sagittal sinus injection (C), and cerebral angiography, lateral view of a right ICA injection in the venous phase (D), showing patency of the stent. Figure is available in color online only. 

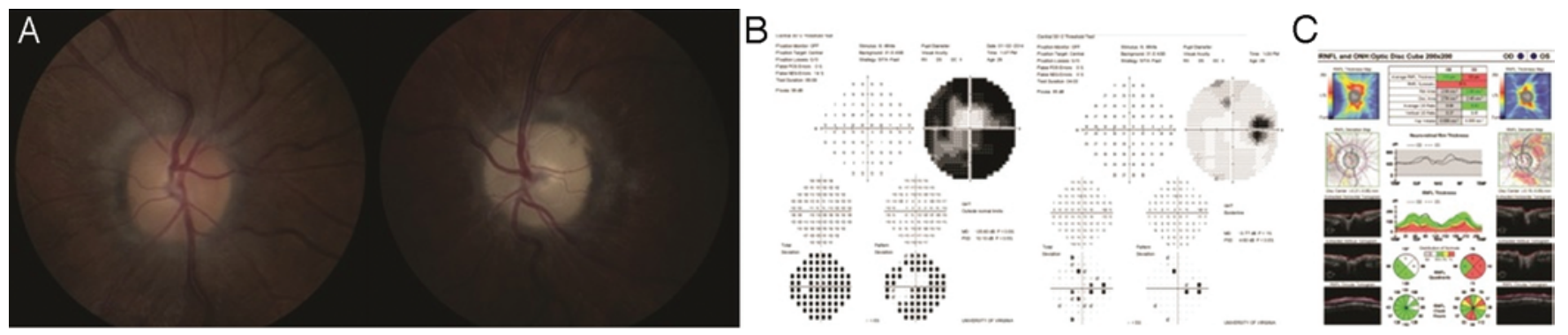

FIG. 5. Case 2. Follow-up ophthalmological examination findings. A: Images from funduscopic examination showing resolution of papilledema. B: Results of visual field testing showing improvement of the bilateral arcuate defects. C: Results of optical coherence tomography showing resolution of nerve fiber thickening. Figure is available in color online only.

dent ophthalmological and neurological assessment and follow-up. Not only did patients experience significant subjective improvements in visual function, but improvements were also demonstrated with rigorous objective assessments. Five of 10 patients had improvement in visual acuity, but this finding may be limited by the subjective nature of testing. ${ }^{21}$ Patients presenting with papilledema had resolution as demonstrated on funduscopic imaging and OCT and improvement on visual field testing. Patients presenting with optic nerve atrophy had optic nerve thinning on follow-up OCT, but improvement in visual field testing. Assessment in a larger cohort of patients is necessary to fully define the patients likely to benefit from stenting. Preliminary evidence demonstrates that even patients with long-standing disease and optic nerve atrophy may have overall benefits in improved visual fields. Further long-term follow-up is necessary as it may be helpful in the prediction or identification of patients at risk for treatment failure.

Further studies are indicated to determine the pathophysiology of IIH in the setting of venous stenosis. ${ }^{6,13,18} \mathrm{In}$ selected patients it remains unclear whether the venous sinus stenosis is the cause of the disease or a secondary consequence of raised ICP; $5,6,22$ as such, IIH remains an acceptable diagnosis, as it remains unknown whether this treatment provides a therapy for the primary cause or a secondary treatment option in an analogous fashion as CSF shunting. ${ }^{5,10,23}$ Additionally, the mechanism by which venous sinus stenting reduces ICP has not been fully elucidated. ${ }^{23}$ It is possible that by increasing the caliber of the transverse sinus, venous outflow is optimized, thereby decreasing global venous congestion in the brain parenchyma. ${ }^{7}$ In addition, decreasing the intraluminal pressure in the intracranial venous system theoretically increases the transluminal pressure differential between the subarachnoid space and the venous system in the arachnoid granulations, thus facilitating or restoring the passive process of CSF absorption into the blood. ${ }^{18}$

Additional studies, including long-term outcome assessments, are indicated in patients with venous sinus stenosis and those undergoing stenting for venous sinus stenosis in the setting of IIH.6,22 Typically, venous stents have a low risk of in-stent stenosis because the venous system is subjected to relatively low pressures and is not as susceptible to atherosclerotic changes as the arterial system. However, venous stents are susceptible to stent-adjacent stenosis. ${ }^{2,9,23}$
In the current series, 2 patients developed stent-adjacent stenosis, and both developed significant pressure gradients across the stenosis requiring repeat treatment. Although this is an uncommonly reported outcome, other studies have reported similar rates of stent-adjacent stenosis. ${ }^{2,9,23}$ Further long-term studies and improved imaging modalities are needed to provide a better understanding of the pathophysiology and optimal treatment options for affected patients.

This study is limited by its small cohort size, which precludes statistical analysis for predictors of treatment failure and complications. Additionally, the study was performed at a single institution, which subjects it to the selection biases and referral patterns of such, as well as to those of the treating physicians. Furthermore, we were unable to compare the efficacy of venous sinus stenting to CSF shunting or optic nerve sheath fenestration, due to the lack of a comparable cohort of medically refractory IIH patients who underwent traditional surgical intervention.

\section{Conclusions}

For appropriately selected patients who have medically refractory IIH and who are found to have venous sinus stenosis with an elevated trans-stenosis pressure gradient and ICP, venous sinus stenting results in immediate abolition of the pressure gradient, reduction in ICP, and functional, neurological, and ophthalmological improvement. Because patients may be at risk for stent-adjacent stenosis, long-term radiographic follow-up is warranted. It is currently unknown whether venous sinus stenosis is the cause of IIH or rather an epiphenomenon of increased ICP. Future prospective comparisons of venous sinus stenting to CSF shunting for medically refractory IIH are necessary to determine optimal therapeutic management.

\section{References}

1. Acheson JF: Idiopathic intracranial hypertension and visual function. Br Med Bull 79-80:233-244, 2006

2. Ahmed RM, Wilkinson M, Parker GD, Thurtell MJ, Macdonald J, McCluskey PJ, et al: Transverse sinus stenting for idiopathic intracranial hypertension: a review of $52 \mathrm{pa}-$ tients and of model predictions. AJNR Am J Neuroradiol 32:1408-1414, 2011

3. Albuquerque FC, Dashti SR, Hu YC, Newman CB, Teleb M, McDougall CG, et al: Intracranial venous sinus stenting for benign intracranial hypertension: clinical indications, tech- 
nique, and preliminary results. World Neurosurg 75:648652, 592-595, 2011

4. Arac A, Lee M, Steinberg GK, Marcellus M, Marks MP: Efficacy of endovascular stenting in dural venous sinus stenosis for the treatment of idiopathic intracranial hypertension. Neurosurg Focus 27(5):E14, 2009

5. Bono F, Giliberto C, Mastrandrea C, Cristiano D, Lavano A, Fera F, et al: Transverse sinus stenoses persist after normalization of the CSF pressure in IIH. Neurology 65:1090-1093, 2005

6. Bono F, Lupo MR, Lavano A, Mangone L, Fera F, Pardatscher K, et al: Cerebral MR venography of transverse sinuses in subjects with normal CSF pressure. Neurology 61:1267-1270, 2003

7. Bono F, Quattrone A: Clinical course of idiopathic intracranial hypertension with transverse sinus stenosis. Neurology 81:695, 2013

8. Donaldson JO: Pathogenesis of pseudotumor cerebri syndromes. Neurology 31:877-880, 1981

9. Ducruet AF, Crowley RW, McDougall CG, Albuquerque FC: Long-term patency of venous sinus stents for idiopathic intracranial hypertension. J Neurointerv Surg 6:238-242, 2014

10. Durst CR, Ornan DA, Reardon MA, Mehndiratta P, Mukherjee S, Starke RM, et al: Prevalence of dural venous sinus stenosis and hypoplasia in a generalized population. J Neurointerv Surg [epub ahead of print], 2016

11. Fields JD, Javedani PP, Falardeau J, Nesbit GM, Dogan A, Helseth EK, et al: Dural venous sinus angioplasty and stenting for the treatment of idiopathic intracranial hypertension. J Neurointerv Surg 5:62-68, 2013

12. Fishman RA: The pathophysiology of pseudotumor cerebri. An unsolved puzzle. Arch Neurol 41:257-258, 1984

13. Friedman DI, Jacobson DM: Diagnostic criteria for idiopathic intracranial hypertension. Neurology 59:1492-1495, 2002

14. Higgins JN, Cousins C, Owler BK, Sarkies N, Pickard JD: Idiopathic intracranial hypertension: 12 cases treated by venous sinus stenting. J Neurol Neurosurg Psychiatry 74:1662-1666, 2003

15. Higgins JN, Owler BK, Cousins C, Pickard JD: Venous sinus stenting for refractory benign intracranial hypertension. Lancet 359:228-230, 2002

16. Higgins JN, Pickard JD: Lateral sinus stenoses in idiopathic intracranial hypertension resolving after CSF diversion. Neurology 62:1907-1908, 2004

17. Kumpe DA, Bennett JL, Seinfeld J, Pelak VS, Chawla A, Tierney M: Dural sinus stent placement for idiopathic intracranial hypertension. J Neurosurg 116:538-548, 2012

18. McGeeney BE, Friedman DI: Pseudotumor cerebri pathophysiology. Headache 54:445-458, 2014

19. Ogungbo B, Roy D, Gholkar A, Mendelow AD: Endovascular stenting of the transverse sinus in a patient presenting with benign intracranial hypertension. Br J Neurosurg 17:565568,2003
20. Radhakrishnan K, Ahlskog JE, Garrity JA, Kurland LT: Idiopathic intracranial hypertension. Mayo Clin Proc 69:169180,1994

21. Radvany MG, Solomon D, Nijjar S, Subramanian PS, Miller NR, Rigamonti D, et al: Visual and neurological outcomes following endovascular stenting for pseudotumor cerebri associated with transverse sinus stenosis. J Neuroophthalmol 33:117-122, 2013

22. Riggeal BD, Bruce BB, Saindane AM, Ridha MA, Kelly LP, Newman NJ, et al: Clinical course of idiopathic intracranial hypertension with transverse sinus stenosis. Neurology 80:289-295, 2013

23. Starke RM, Wang T, Ding D, Durst CR, Crowley RW, Chalouhi N, et al: Endovascular treatment of venous sinus stenosis in idiopathic intracranial hypertension: complications, neurological outcomes, and radiographic results. ScientificWorldJournal 2015:140408, 2015

24. Thurtell MJ, Wall M: Idiopathic intracranial hypertension (pseudotumor cerebri): recognition, treatment, and ongoing management. Curr Treat Options Neurol 15:1-12, 2013

25. Wall M, McDermott MP, Kieburtz KD, Corbett JJ, Feldon SE, Friedman DI, et al: Effect of acetazolamide on visual function in patients with idiopathic intracranial hypertension and mild visual loss: the idiopathic intracranial hypertension treatment trial. JAMA 311:1641-1651, 2014

\section{Disclosures}

The authors report no conflict of interest concerning the materials or methods used in this study or the findings specified in this paper.

\section{Author Contributions}

Conception and design: Starke, Newman. Acquisition of data: all authors. Analysis and interpretation of data: Starke, Liu, Durst, Wang, Ding, Newman. Drafting the article: all authors. Critically revising the article: all authors. Reviewed submitted version of manuscript: all authors. Approved the final version of the manuscript on behalf of all authors: Starke. Statistical analysis: Starke, Newman. Administrative/technical/material support: all authors. Study supervision: Starke, Liu, Durst, Crowley, Newman.

\section{Supplemental Information}

\section{Current Affiliations}

Dr. Crowley: Department of Neurological Surgery, Rush University Medical Center, Chicago, IL.

\section{Correspondence}

Robert M. Starke, University of Miami, Department of Neurological Surgery \& Radiology, 1475 NW 12th Ave., C212, Box 356340, Miami, Florida 33136. email: bobby.starke@gmail.com. 